\title{
CRYPTOCOCCOSIS OF THE HEART
}

\author{
BY \\ IDRIS JONES, EUGEN NASSAU, AND PETER SMITH
}

From Harefield Hospital, Harefield, Middlesex

Disseminated cryptococcosis is now a well-recognized clinical and pathological entity (Littman, 1959). The infection is believed to be airborne. The causative organism, Cryptococcus neoformans, is a pathogenic yeast occurring as a saprophyte in some soils, and has also been encountered in pigeons' excreta. Primary pulmonary cryptococcosis is often a self-limiting infection. Disseminated cryptococcosis, occasionally following the primary lung infection, is invariably fatal if untreated, and is not infrequently associated with reticuloses in patients with low resistance to infection.

The meningeal and cerebral form of the disease is as a rule responsible for the invariably fatal outcome in untreated cases. No specific mention is made by Littman (1959) or Baker and Haugen (1955) of myocardial involvement in disseminated cryptococcosis. This was the dominant feature of the clinical picture in the case reported here, which presented with severe tachycardia and acute heart failure.

\section{Case Report}

The patient was a man aged 31, who was admitted to Harefield Hospital on January 5, 1961. His complaint was of breathlessness which he had had for two days, and of intermittent palpitations which had been present for four months. The breathlessness at first came on if he walked as little as 100 yards, later coming on during the night in paroxysms, and on the day of admission coming on at rest. He had some cough and during a coughing bout he felt faint. There was nothing relevant in his past history.

Clinical Findings. He was dyspnœic at rest and obviously anxious. The heart was clinically enlarged, the apex being present in the anterior axillary line on percussion but impalpable. The apex rate was uncountable and the pulse was imperceptible. His blood pressure was then 100/80 mm. Hg. The jugular venous pressure was not raised, but he was very tender in the upper abdomen over the enlarged liver. He had moist sounds at both lung bases with occasional rhonchi. The heart sounds were very faint. An electrocardiogram showed paroxysmal ventricular tachycardia with grossly abnormal complexes (Fig. 1A).

He was given $300 \mathrm{mg}$. procainamide intravenously on admission under electrocardiographic control, and this had no effect. He slept all night, and the following morning his heart rate was still over 200 and further procainamide was given to a total of $900 \mathrm{mg}$., without any effect. He was then started on 10 grains of quinidine at 10 p.m., and 5 grains every hour after that, up to a total of 20 grains, at the end of which time his heart rate had reverted to normal rhythm. The apex rate then was 64 with very soft heart sounds, and blood pressure 90/60 mm. Hg. He was given intramuscular mephine because of the low blood pressure. He was kept on a maintenance dose of quinidine grains 3 twice a day. An electrocardiogram made after reversion to normal rhythm showed prolongation of the P-R interval with occasional dropped beats (Fig. 1B). He remained in normal rhythm and his blood pressure remained steady at about $110 / 80 \mathrm{~mm}$. $\mathrm{Hg}$ for the rest of his stay in hospital. A radiograph of his chest (Fig. 2) showed great cardiac enlargement and congested lung fields. A further radiograph taken on January 31, 1961 showed no reduction of the size of the heart, but the pulmonary congestion was less. Though he felt well, his electrocardiogram remained persistently abnormal with a prolonged P-R interval with some dropped beats and inverted $T$ waves in all the $V$ leads.

Course of Illness. He was considered at the time to have a cardiomyopathy and was discharged home on February 6, on amytal grains 1 twice a day, and mycardol tab. 1 three times a day with trinitrin grains 100th to take if he had any chest pain. He was seen as an outpatient at regular intervals after this and his heart rate remained between 60 and 80 with cardiographic evidence of heart block still persisting. 
A

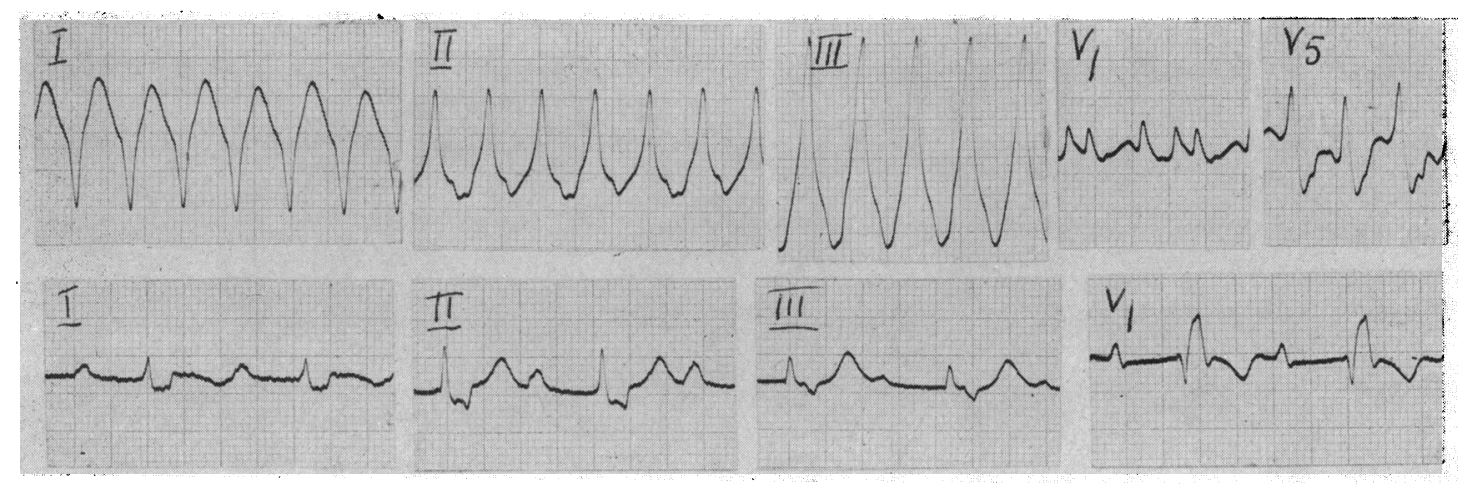

Fig. 1.-(A) Electrocardiogram during an attack, showing paroxysmal ventricular tachycardia with grossly abnormal complexes. (B) Electrocardiogram between attacks, showing prolongation of the $\mathbf{P}-\mathbf{R}$ interval.

He was admitted again on April 10 having had an acute attack of dyspnœa two hours previously. The electrocardiogram again showed ventricular tachycardia, and he was given a total dose of $\mathbf{4 0}$ grains of quinidine during the night, after which he reverted to normal rhythm. He was discharged home 10 days after admission and kept on a maintenance dose of quinidine grains 3 twice a day.

He was readmitted as an emergency on June 1 with sudden onset of sweating and fluttering feelings in his chest. The signs were as before with the heart grossly enlarged and an apex rate over 150, the blood pressure being $85 / 70$. He again reverted to normal rhythm with 20 grains of quinidine, and again he was discharged home taking 5 grains quinidine three times a day and was warned to avoid undue exertion or stress. On July 16, he was found dead in his bed following an acute emotional upset at home.

Necropsy was performed four days after death.

Necropsy. There were marked autolytic changes of all organs. The lungs showed scattered miliary lesions, resembling miliary tubercles. The hilar and

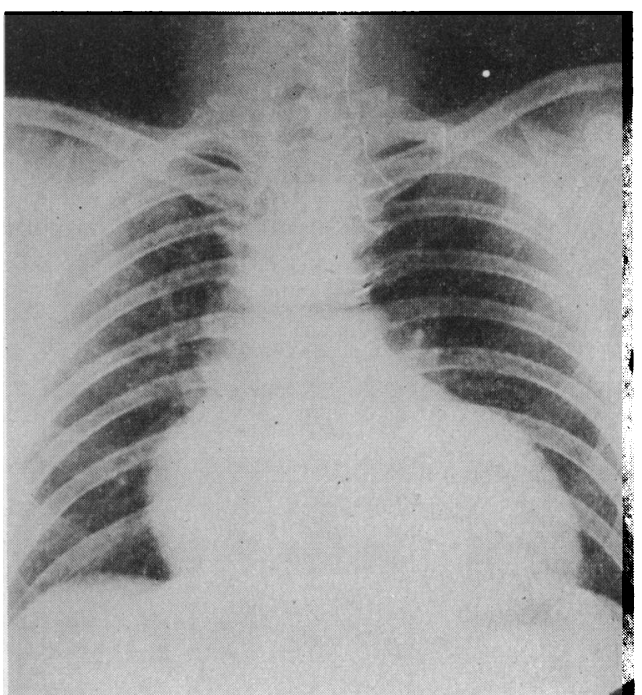

Fig. 2.-Radiograph of chest showing great cardiac enlargement and congested lung fields paratracheal glands contained caseous and partly calcified foci. The pericardium contained several ounces of blood-stained fluid. The heart was enlarged, mainly due to dilatation. The myocardium was flabby and the left ventricle showed greyish streaks, suggestive macroscopically of myocarditis. The coronary arteries appeared normal. Apart from a calcified lymph node at the root of the mesentery there were no abnormal findings in the abdomen. The central nervous system was not examined; in view of the clinical history and necropsy findings centering on the heart, as well as the advanced autolytic changes present, this was not considered essential.

Histological examination confirmed the macroscopical findings as epithelioid and giant cell granulomata in the pulmonary and glandular lesions. Similar granulomata, with variable fibrosis, were present in the myocardium (Fig. 3). Ziehl Neelsen staining failed to demonstrate tubercle bacilli. Sections stained by Gridley's and P.A.S. staining revealed Cryptococcus neoformans in the granulomata in the heart, lungs, and caseous glandular lesions.

\section{Summary}

An account is given of a patient with cryptococcosis of the heart. We can find no previous record of such cardiac involvement. The presentation with acute dyspnœa and tachycardia with 


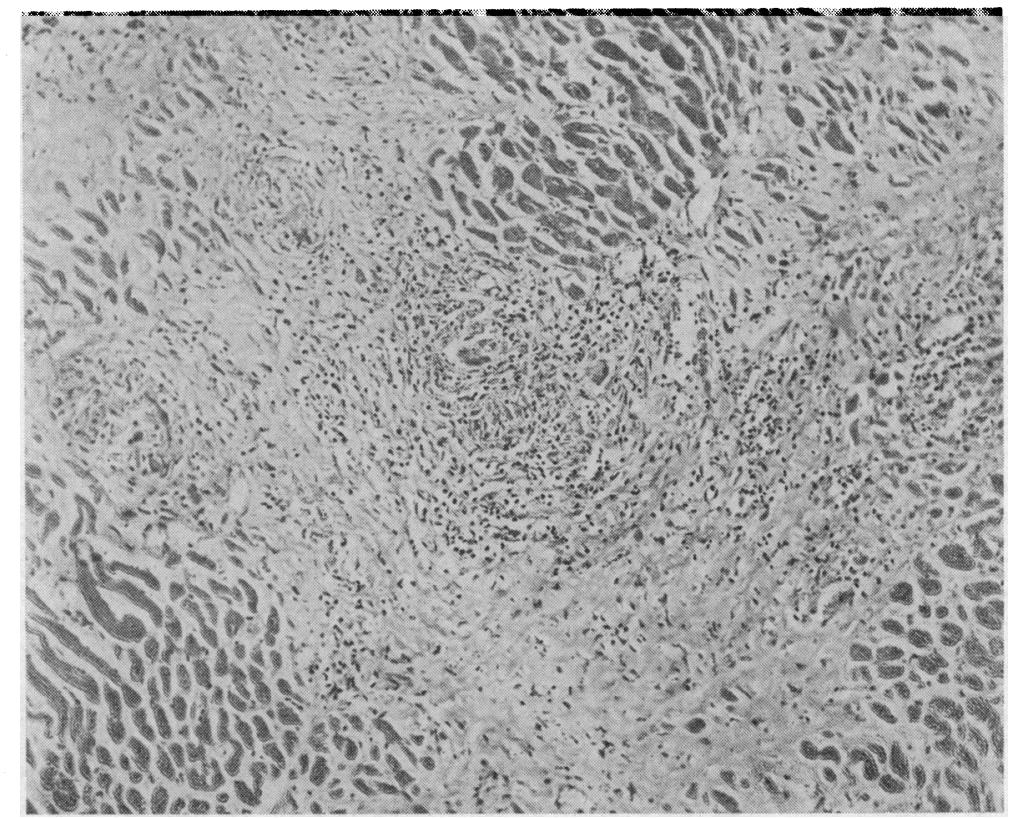

FIG. 3.-Photomicrograph of myocardium showing granulomata, with variable fibrosis.

cardiomegaly and gross electrocardiographic abnormalities in a young man caused us to diagnose cardiomyopathy of unknown ætiology. The final diagnosis was made at necropsy. There were no symptoms or signs during life to suggest disease in any system other than the cardiovascular.

\section{References}

Baker, R. D., and Haugen, R. K. (1955). Tissue changes and tissue diagnosis in cryptococcosis. A study of 26 cases. Amer. J. clin. Path., 25, 14.

Littman, M. L. (1959). Cryptococcosis (torulosis). Current concepts and therapy. Amer. J. Med., $27,976$. 